\title{
PROGNOSTIC SIGNIFICANCE OF MAGNETIC RESONANCE IMAGING IN PATIENTS WITH PROSTATE INTRAEPITHELIAL NEOPLASIA
}

10.36740/WLek202101106

\author{
Maksym P. Melnychuk \\ STATE INSTITUTION OF SCIENCE'RESEARCH AND PRACTICAL CENTER OF PREVENTIVE AND CLINICAL MEDICINE'STATE ADMINISTRATIVE DEPARTMENT, \\ KYIV, UKRAINE
}

\begin{abstract}
The aim: To determine prognostic significance of mpMRI in prostate intraepithelial neoplasia (PIN) diagnostics.

Materials and methods: The results of examination of 52 patients with PIN were assessed in mpMRI using PIRADS criteria. The total number of samples with PIN amounted 166. According to PIRADS MRI assessment of central and peripherial zones was made separately. The use of T2WI, DWI, DCE in patients with high grade and low grade PIN was studied. MRI was performed before prostate biopsy (MRI cognitive fusion biopsy). During 3-year follow-up rebiopsies were performed with prostate cancer detection. PIRADS values of PIN lesions with malignisation were compared with those without following tumor transformation.

Results: There was a difference in values of PIRADS characteristics between PIN and benign prostatic tissue. The mean of PIRADS gradation in samples with PIN was 2,1. Among them 47 (28,3\%) PIN samples had gradation 3 (the presence of clinically significant cancer is equivocal), in 8 (4,8 \%) cases - gradation 4 (clinically significant cancer is likely to be present). The mean of PIRADS gradation was in $24 \%$ larger in cases with subsequent PC detection than in cases without malignisation.

Conclusions: MRI parameters in PIN cases differ from normal prostate tissue. PIRADS assessment has prognostic significance of following malignisation of PIN pieces that have similar properties on MRI as prostate cancer. Further study is required to stratify all PIN patients into groups of high malignisation risk in order to perform detailed examination and treatment.
\end{abstract}

KEY WORDS: prostate intraepithelial neoplasia, multiparametric MRI

Wiad Lek. 2021;74(1):35-38

\section{INTRODUCTION}

Prostate intraepithelial neoplasia (PIN) is considered to be a precancerous lesion and is of great medical and social importance. According to WHO (world health organization) data the incidence of prostate cancer (PC) in the world is about 1100000 new cases, besides PC mortality is about 300000 [1]. One of the ways to improve early diagnostics and treatment results is timely detection of precancerous processes, their study and development of diagnostics, treatment and prognosis algorithm in patients with precancerous diseases [2,3]. Such high informative method as magnetic resonance imaging (MRI) for a long time is used in PC diagnostics. Technical improvement of MRI with use of multiparametric methods has improved its sensitivity and specificity [4].

Structural MRI parameters charactirise anatomic peculiarities (T2), that are combined with functional parameters (DWI - diffusion-weighted imaging, contrast inhancement) to detect biological tumour properties [5]. Using together these methods allow to assess simultaneously different tissue properties, to determine zonal prostate anatomy and to define vascularisation rate of prostate neoplasms [6,7].
At the same time diagnostic role of MRI in patients with PIN is determined insufficiently. Considering clinical significance of PIN as a precancerous state it is actual to define specific MRI features of PIN for early diagnostics and prevention of malignisation [8]. Systematization and instrumental data comparison are essential requirements for detection of specific MRI properties of precancerous processes. Prognosis of clinical course of PIN and its malignant potential is based on pathomorphology and immunohystochemistry data. The use of mpMRI for determination of high malignisation risk group among patients with PIN aims to further detailed examination of patients with rebiopsy and special treatment.

\section{THE AIM}

To study informativeness of mpMRI in prostate intraepithelial neoplasia diagnostics and prognostication of malignant transformation.

\section{MATERIALS AND METHODS}

We studied 52 patients from 55 to 73 years old (mean age $66 \pm 2,3$ y.o.) who underwent transrectal multifocal prostate 
biopsy under trasrectal ultrasonic guidance diagnosing PIN. 34 patients had peripheral zone PIN, 11 patients had central zone PIN and 7 patients had both peripheral and central zone PIN. MRI was perfomed before prostate biopsy. Indication to biopsy was PC suspicion based on PSA, digital rectal examination, ultrasonography. Then cognitive fusion MRI targeted biopsy was perfomed.

For systematization of targeted pieces was used prostate segmentation according to PIRADS (prostate imaging reporting and data system) concept that is adaptation of EAU 2012 card and recommendations of European Society of Urogenital Radiology ESUR based on anatomical and histological prostate structure.

MpMRI was perfomed with the help of device Hitachi Echelon with magnetic field capacity 1,5 T. MRI assessment was made according to international PIRADS system separately for peripheral and central zone. In complex evaluation such MRI parameters as T2, DWI and dynamic contrast enhancement (DCE) were used.

Using $\mathrm{T} 2$ regimen we determined heterogenity and local changes defining their size, quantity, zonal localization. Suspicious prostate lesions had depressed signal intensity in T2WI with unequal contour, low water molecule diffusion (high DWI signal). With the help of DCE we determined the quick contrast accumulation and contrast evacuation and subsequent contrast accumulation by surrounding parenchyma.

Findings were estimated according to PIRADS with PC probability, PC aggressiveness, localization and extraprostatic spread. Local changes of prostate tissue were defined and detailed with the need of morphological confirmation. After that MRI data were compared with pathohistological findings of corresponding prostate lesions.

As part of patients had PIN in more than one histological pieces, we analized total amount of lesions that contained PIN. The most often PIN was localized in 1-2 pieces, namely in $21(41 \%)$ of patients (among them in 1 piece in 7 patients, in 2 pieces in 14 cases). 20 patients (38\%) had PIN in 3-4 specimens (among them in 3 pieces in 11 patients, in 4 pieces in 9 patients). In 11 (21\%) cases PIN was detected in 5 and more specimens (among them in 5 pieces -6 patients, in 6 pieces - in 3 patients, in 7 pieces in 2 patients). Total amount of PIN specimens in patients whom was perfomed cognitive fusion MRI biopsy was 166. Among them were 104 specimens of peripheral zone PIN and 62 specimens of central zone PIN.

By evaluation of PIN specimens according to PIRADS criteria were used grades of clinical significant PC risk. 1 grade corresponded to very low (clinically significant cancer is highly unlikely to be present), 2 grade: low (clinically significant cancer is unlikely to be present), 3 grade: intermediate (the presence of clinically significant cancer is equivocal), 4 grade: high (clinically significant cancer is likely to be present), 5 grade: very high (clinically significant cancer is highly likely to be present).

In order to detect PC and to determine prognostic significance of mpMRI rebiopsies with 6 months interval during 3 years were perfomed. MpMRI data of each specimen were compared with histological data of corresponding pieces obtained after rebiopsy.

All described research methods were approved by ethic commission of State institution of science 'Research and practical center of preventive and clinical medicine' State administrative department. All patients gave an informed concent before involving into investigation.

\section{RESULTS}

MpMRI data of peripheral prostate zone were studied. Analysis T2WI of 104 PIN specimens established the following results. In $24(23 \%)$ of PIN cases was observed grade 1 (PIRADS), in $42(40,4 \%)$ PIN lesions was determined grade 2 , in $33(31,7 \%)$ PIN pieces was detected grade 3 and $5(4,8 \%)$ of PIN specimens had grade 4 . In addition, there were no grade 5 data in peripheral zone PIN.

The mean value according to PIRADS in peripheral zone PIN amounted 2,2 (figure 1).

MpMRI data of central prostate zone were studied. Analysis T2WI of 62 PIN specimens established the following results. In $17(27,4 \%)$ of PIN cases was observed grade 1 (PIRADS), in 26 (42\%) PIN lesions was determined grade 2 , in 19 (30,1 \%) PIN pieces was detected grade 3. Besides, there were no grade 4 and grade 5 data in central zone PIN.

The mean value according to PIRADS in central zone PIN amounted 2 (figure 2).

Functional parameters of 166 PIN lesions were assessed by studiing of DWI in mpMRI. Analysis DWI of 166 PIN specimens established the following results. In $63(38 \%)$ of PIN cases was observed grade 1 (PIRADS), in 65 (39\%) PIN lesions was determined grade 2, in $32(19,3 \%)$ PIN pieces was detected grade 3 and $6(3,6 \%)$ of PIN specimens had grade 4 . In addition, there were no grade 5 data in DWI in patients with PIN. The mean DWI value according to PIRADS amounted 1,7 (figure 3).

Dynamic MRI with contrast enhancement report was negative (no early enhancement, or diffuse enhancement not corresponding to a focal finding on $\mathrm{T} 2 \mathrm{~W}$ and/or DWI or focal enhancement corresponding to a lesion demonstrating features of benign prostatic hyperplasia on T2WI) or positive (focal, and earlier than or contemporaneously with enhancement of adjacent normal prostatic tissues, and corresponds to suspicious finding on T2 and/or DWI). DCE data were not used in general value in cases of low PC probability (grades 1 and 2) or high PC probability (grades 4 and 5). In cases of PIRADS 3 (in T2WI, DWI) local contrast enhancement in DCE increased the grade to PIRADS 4.

As a result of complex mpMRI (T2WI, DWI, DCE) analysis of all peripheral and central PIN specimens was developed general value according to PIRADS criteria.

The following general values mpMRI among 166 PIN specimens were determined: in $48(28,9 \%)$ cases was defined grade 1, in 63 (38\%) lesions - grade 2, in $47(28,3$ $\%)$ - grade 3 , in $8(4,8 \%)$ specimens - grade 4 . There were no grade 5 value among PIN patients determined (fig. 4). The mean PIRADS value in PIN was 2,1. 


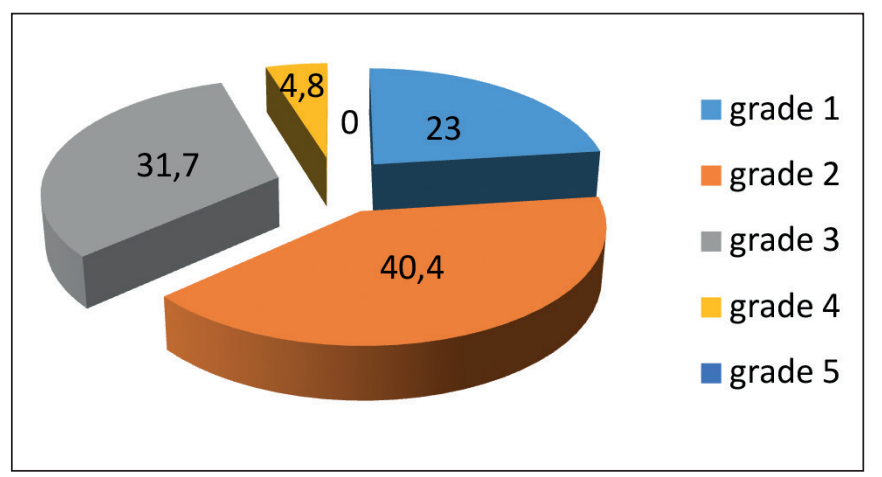

Fig. 1. Structure of T2Wl in peripheral zone PIN values according to PIRADS

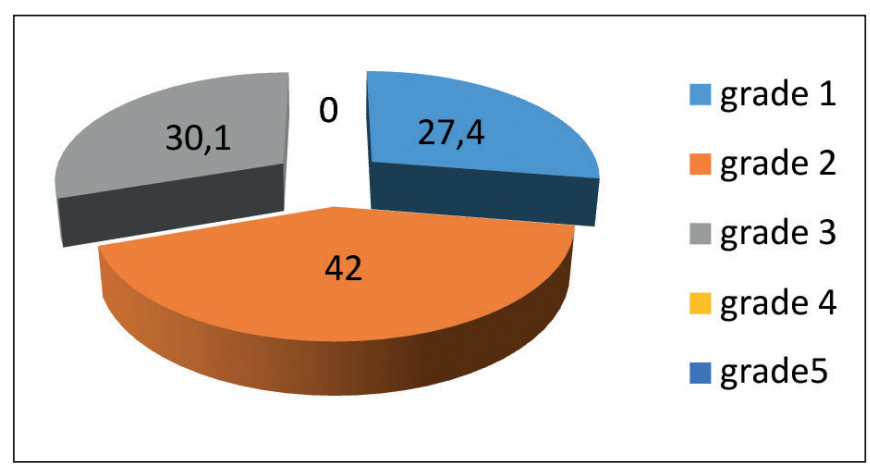

Fig. 2. Structure of T2WI in central zone PIN values according to PIRADS

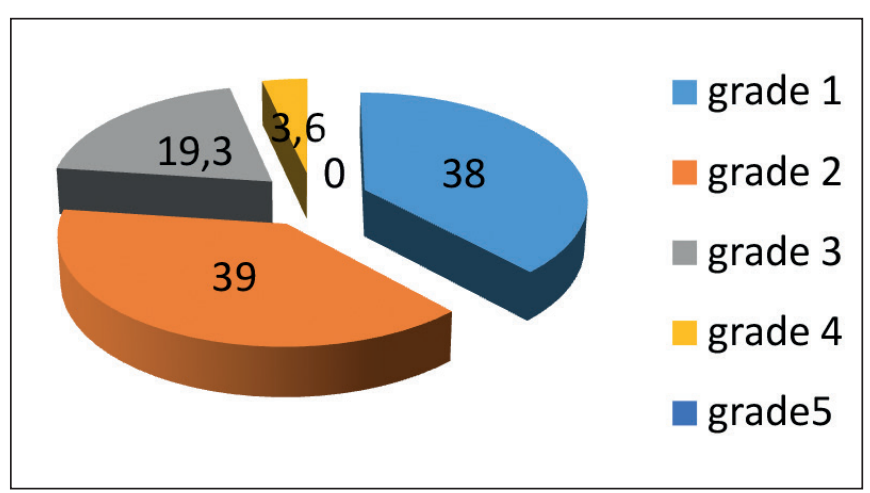

Fig.3. Structure of DWI of PIN lesions according to PIRADS

On the basis of obtained data we came to the conclusion that the situation of mpMRI in patients with PIN is heterogeneous. Though the majority of PIN specimens, namely $67 \%$ has benign tissue attributes (grades 1-2), exists a part of lesions, namely $33 \%$, which has PC suspicion (grades 3-4). These results had to be detailized to define the group of patients with high risk of malignant transformation of PIN to PC.

During 3-year follow-up with 6 months interval prostate rebiopsies were performed. The specimens with cancer detection in correlation with primary mpMRI data were analized. It was determined that during 3-year follow up of 52 patients who took part in the study PC was diagnosed in 14 men. The total amount of specimens with PC was 45 .

The colletion of mpMRI data with corresponding $45 \mathrm{ma}-$ lignant prostate lesions determined the following PIRADS grades: in $5(11 \%)$ of cases was detected grade 1 , in $15(33,3$

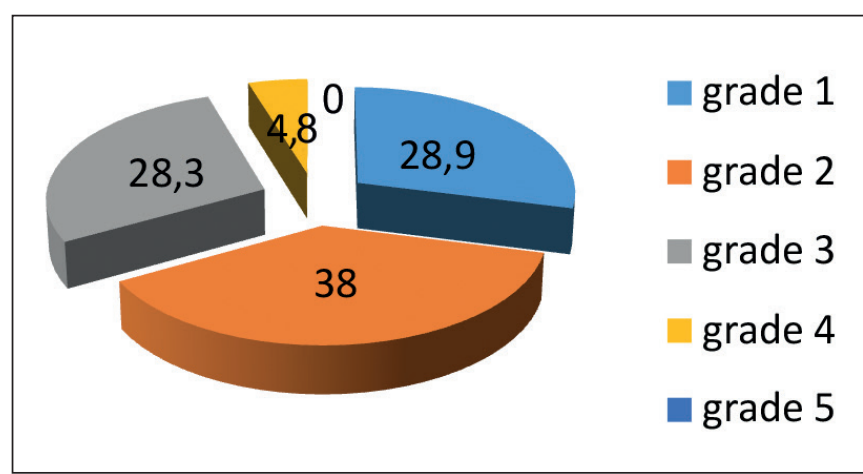

Fig. 4. Structure of PIN lesions according to PIRADS.

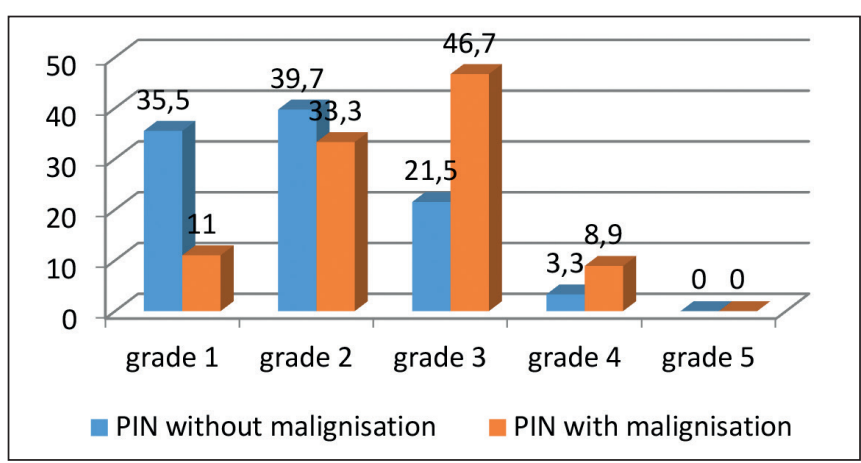

Fig. 5. Distribution of PIRADS grades in PIN specimens corresponding to malignisation

\%) lesions was observed grade 2 , in $21(46,7 \%)$ specimens - grade 3 , in $4(8,9 \%)$ was determined grade 4 . There were no grade 5 value among PIN patients determined (fig. 5).

The mean PIRADS value of PIN specimens with subsequent malignisation was 2,5 and was in $24 \%$ bigger than in PIN specimens without subsequent malignisation.

\section{DISCUSSION}

Obtained results confirm role of PIN in prostate cancerogenesis as transitional state from normal to malignant tissue. Moreover mpMRI data assessed in T2WI, DWI and DCE regimens showed the differences between normal prostate tissue and PIN.

Based on obtained data we came to the conclusion that mpMRI plays an important role not only in PC but also in PIN. Although PIN has no specific MRI signs, PIN specimens are often visualized as suspicious for PC and have common characteristics with adenocarcinoma. Prognostic significance has PIRADS grade, that is complex evaluation of MRI parameters describing morphological picture of prostate lesions. Direct correlation between malignant transformation potential and PIRADS rate was determined.

As a result technical possibilities of MRI contribute to investigation of morphological structure of prostate lesions, namely PIN and PC. Methodology, indications, technical aspects, interpretation of MRI data need futher studiing in order to improve sensitivity and specificity of prostate biopsy. 


\section{CONCLUSIONS}

According to mpMRI data PIN occupies intermediate place between benign prostate tissue and PC. The mean rate by PIRADS criteria in PIN was 2,1. PIRADS grade has prognostic significance for PIN malignisation. The mean PIRADS grade in PIN specimens with subsequent PC ranged 2,5 and was in $24 \%$ bigger than in PIN specimens without subsequent malignisation. Multiparametric MRI is a sensitive diagnostic method and can be used for detection of patients with high malignisation risk.

\section{REFERENCES}

1. Heidenreich A., Abrahamsson P., Artibani W. et al. Early detection of prostate cancer. European Association of Urology recommendation. Eur Urol. 2013; 64(3):347-354.

2. Jung S-H., Shin S., Kim M. et al. Genetic Progression of High Grade Prostatic Intraepithelial Neoplasia to Prostate Cancer. European Urology. 2016; 69 (5):831-833.

3. Donghyun L., Chunwoo L., Taekmin K. Clinical features and prognosis of prostate cancer with high-grade prostatic intraepithelial neoplasia. Korean J Urol. 2015; 56(8):565-571.

4. Brawer M., Lange P. Prostate-specific antigen and premalignant change: implications for early detection CA. Cancer J Clin. 1989; 39:361-375.

5. Dwivedi D., Kumar R., Bora G. Multiparametric MR can identify high grade prostatic intraepithelial neoplasia (HGPIN) lesions and predict future detection of prostate cancer in men with a negative initial prostate biopsy. Magn Reson Imaging. 2016; 34(8):1081-1086.

6. Woodrum D., Gorny K., Greenwood B. MRI-Guided Prostate Biopsy of Native and Recurrent Prostate Cancer. Semin Intervent Radiol. 2016; 33:196-205.

7. Dwivedi K., Rajeev K., Girdhar S. Multiparametric MR can identify high grade prostatic intraepithelial neoplasia (HGPIN) lesions and predict future detection of prostate cancer in men with a negative initial prostate biopsy. Magnetic Resonance Imaging. 2016; 34:1081-1086.

8. Borkowetz A., Platzek I., Toma M., Laniado M. Comparison of systematic transrectal biopsy to transperineal magnetic resonance imaging/ ultrasound-fusion biopsy for the diagnosis of prostate cancer. BJU Int. 2015; 116:873-879.
The study was made as a part of research work 'Optimisation of specialized and high specialized surgical treatment based on the 'Fast treck surgery' in thyroid gland, parathyroid gland, nasopharynx, internal and reproductive organs, abdominal wall, blood vessels and joints diseases using atom-power microscopy with application of polarizing mixture for implants processing', which is performed in State institution of science 'Research and practical center of preventive and clinical medicine' State administrative department.

\section{ORCID and contributionship:}

Maksym P. Melnychuk: 0000-0003-1194-3496 ${ }^{\text {A,B,C,D,E,F }}$

\section{Conflict of interest:}

The Author declare no conflict of interest.

\section{CORRESPONDING AUTHOR Maksym P. Melnychuk}

State institution of science' Research and practical center of preventive and clinical medicine'

State administrative department 10 Bazhana Avenue, apt. 115, 02140 Kyiv, Ukraine tel: +380975637943

e-mail: maksymmelnychulk1980@gmail.com

Received: 05.03 .2020

Accepted: 10.10 .2020

A - Work concept and design, B - Data collection and analysis, C - Responsibility for statistical analysis, $\mathbf{D}$-Writing the article, $\mathbf{E}$ - Critical review, $\mathbf{F}$ - Final approval of the article 